\title{
Integration Formulae Involving Derivatives
}

\author{
By T. N. L. Patterson*
}

\begin{abstract}
A method, developed by Hammer and Wicke, for deriving high precision integration formulae involving derivatives is modified. It is shown how such formulae may be simply derived in terms of well-known polynomials.
\end{abstract}

1. Introduction. The construction of high precision integration formulae which make use of the derivatives of the integrand has been discussed by Stroud and Stancu [1] and by Hammer and Wicke [2]. Stroud and Stancu [1] considered formulae of the form

$$
\int_{a}^{b} w(x) f(x) d x=\sum_{j=1}^{n} \sum_{i=0}^{k j-1} H_{j}^{(i)} f^{(i)}\left(x_{j}\right)
$$

and have calculated a few results for the special case, $k_{j}=k$, for all $j$, with $n=1(1) 7$, $k=3$ and 5 and $w(x)=1, e^{-x^{2}}$ and $e^{-x}$. The formulae have degree $n(k+1)-1$, use $n k$ functional evaluations and are obtained by solving sets of nonlinear equations.

Hammer and Wicke [2] considered formulae of the form

$$
\int_{-1}^{1} f(x) d x=2 \sum_{i=0}^{[(k-1) / 2]} f^{(2 i)}(0) /(2 i+1) !+\sum_{j=1}^{m} a_{j}\left[f^{(k)}\left(x_{j}\right)-f^{(k)}\left(-x_{j}\right)\right]
$$

where $[x]$ denotes the largest integer $\leqq x$. These formulae have degree $4 m+k$ when $k$ is odd and $4 m+k-1$ when $k$ is even and use $2 m+1+[(k-1) / 2]$ function values. The $m$ abscissae $x_{j}$ are the zeros of a numerically determined orthogonal polynomial. Struble [3] has calculated formulae for the cases $k=1$ and 2 and $m=1(1) 10$. He notes that some numerical difficulties occur for large values of $m$. The formulae of Stroud and Stancu [1] use about twice as many function values as the Hammer and Wicke [2] formulae for the same integrating degree and are much more difficult to obtain.

This paper is concerned with formulae of the Hammer and Wicke type. It is shown that with a slight decrease in integrating power the derivation of the formula can be simplified and some results are presented.

2. Theory. The formulae of Hammer and Wicke [2] are based on the well-known result that

$$
\int_{0}^{1}\left(\int_{0}^{x}\right)^{n} g(x)(d x)^{n+1}=\frac{1}{n !} \int_{0}^{1}(1-x)^{n} g(x) d x
$$

where $\left(\int_{0}^{x}\right)^{n} g(x)(d x)^{n}$ denotes repeated integration over $[0, x]$.

It is equally true that

Received June 17, 1968.

* This research has been supported by the National Aeronautics and Space Administration under Contract NsG-269. 


$$
\int_{-1}^{1}\left(\int_{-1}^{x}\right)^{n} g(x)(d x)^{n+1}=\frac{1}{n !} \int_{-1}^{1}(1-x)^{n} g(x) d x .
$$

It is straightforward to show by repeated integration of $f^{(k)}(x)$ that,

$$
\int_{-1}^{1}\left(\int_{-1}^{x}\right)^{k} f^{(k)}(x)(d x)^{k+1}=\int_{-1}^{1} f(x) d x-\sum_{i=0}^{k-1} \frac{2^{i+1}}{(i+1) !} f^{(i)}(-1) .
$$

Thus using (4) gives,

$$
\begin{aligned}
\int_{-1}^{1} f(x) d x= & \frac{1}{k !} \int_{-1}^{1}(1-x)^{k} f^{(k)}(x) d x+\sum_{i=0}^{k-1} \frac{2^{i+1}}{(i+1) !} f^{(i)}(-1) \\
= & \frac{1}{k !} \sum_{j=1}^{m} H_{j} f^{(k)}\left(x_{j}\right)+\sum_{i=0}^{k-1} \frac{2^{i+1}}{(i+1) !} f^{(i)}(-1) \\
& \quad+\frac{2^{k+2 m+1}}{(k+2 m+1)(2 m) ! k !}\left[\frac{m !(k+m) !}{(k+2 m) !}\right]^{2} f^{(2 m+k)}(\eta) .
\end{aligned}
$$

In the remainder term $\eta$ lies in $[-1,1]$. It is clear that the best possible accuracy will be obtained by integrating the first term on the right-hand side of (6) using a quadrature formula of highest precision with respect to the weight function $(1-x)^{k}$ over $[-1,1]$. The abscissae, $x_{j}$, of this quadrature formula are simply the roots of the Jacobi polynomial $P_{m}^{(k, 0)}(x)$ (Krylov [4]) and the weights $H_{j}$ are given by

$$
H_{j}=\frac{2^{k+1}}{\left(1-x_{j}\right)^{2}\left[P_{m}{ }^{(k, 0)}\left(x_{j}\right)\right]^{2}} .
$$

The resulting quadrature formula (7) has degree $2 m+k-1$ and uses $m+k$ functional evaluations. For the same integrating degree (7) uses about $k / 2$ more functional evaluations than (2). Tables of the abscissae $x_{j}$ and weights $H_{j}$ have been given by Stroud \& Secrest [5] for $k=1$ using 2(1)30 points and for $k=2,3$ and 4 using 2(1)20 points.

Southwest Center for Advanced Studies Dallas, Texas 75230

1. A. H. Stroud \& D. D. Stancu, "Quadrature formulas with multiple Gaussian modes," $J$. Soc. Indust. Appl. Math., Ser. B, Numer. Anal., v. 2, 1965, pp. 129-143. MR 31 \#177.

2. P. C. HAMmer \& H. H. WICKE, "Quadrature formulas involving derivatives of the integrand," Math. Comp., v. 14, 1960, pp. 3-7. MR $22 \# 1073$.

3. G. W. STRUBLE, "Tables for use in quadrature formulas involving derivatives of the integrand," Math. Comp., v. 14, 1960, pp. 8-12. MR 22 \#1074.

4.' V. I. KRYLov, A pproximate Calculation of Integrals, Fizmatgiz, Moscow, 1959; English transl., Macmillan, New York, 1962. MR 22 \#2002; MR 26 \#2008.

5. A. H. STroud \& D. Secrest, Gaussian Quadrature Formulas, Prentice-Hall, Englewood Cliffs, N. J., 1966, 374 pp. MR 34 \#2185. 